\title{
Influence of aortitis on late outcomes after repair of ascending aortic aneurysms
}

\author{
Hirokazu Fujimoto, MD, PhD, ${ }^{\text {a }}$ Meghana R. K. Helder, MD, ${ }^{\text {a }}$ Alberto Pochettino, MD, ${ }^{a}$ \\ Kevin L. Greason, MD, ${ }^{a}$ Rakesh M. Suri, MD, ${ }^{a}$ Richard C. Daly, MD, ${ }^{a}$ Joseph A. Dearani, MD, ${ }^{a}$ \\ Joseph J. Maleszewski, MD, ${ }^{\mathrm{b}}$ Zhuo Li, MS, ${ }^{\mathrm{c}}$ and Hartzell V. Schaff, MD ${ }^{\mathrm{a}}$
}

\begin{abstract}
Objective: To determine outcomes of repair of ascending aortic aneurysms in patients with histopathologic diagnoses of aortitis.

Methods: We reviewed histopathologic findings and outcomes of elective repair of ascending aortic aneurysms between January 1, 1955, and December 31, 2012. Noninfectious aortitis was identified in 186 patients, and we compared outcomes for these patients with outcomes for others operated on at the same time with diagnoses of medial degeneration $(n=317)$ or atherosclerosis $(n=232)$.
\end{abstract}

Results: Early mortality ( $<30$ days postoperatively) for patients with aortitis was $2 \%$, and overall 10 -year survival was $45 \%$, compared with $66 \%$ for patients with medial degeneration, and $45 \%$ for patients with atherosclerosis $(P<.001$ vs medial degeneration). In addition to histopathologic diagnosis, overall mortality was influenced by older age at operation (hazard ratio [HR]: $1.060 ; 95 \%$ confidence interval [CI], 1.046-1.077; $P<.001)$, chronic obstructive pulmonary disease (HR: 1.560 ; 95\% CI: $1.136-2.136 ; P=.006$ ); concomitant coronary artery bypass grafting (HR: 1.980; 95\% CI: 1.520-2.600; $P<.001$ ); and use of circulatory arrest (HR: $1.500 ; 95 \%$ CI: $1.148-1.960 ; P=.003$ ). Risk of aortic reoperation at 10 years was $21 \%$ for aortitis patients, compared with $11 \%$ for those with medial degeneration, and $19 \%$ for patients with atherosclerosis $(P=.028)$.

Conclusions: Patients with repaired ascending aneurysms secondary to noninfectious aortitis have low early mortality, but late risks of death and aortic reoperation are increased, compared with these outcomes for patients with aneurysms that result from medial degeneration. (J Thorac Cardiovasc Surg 2015;150:589-94)

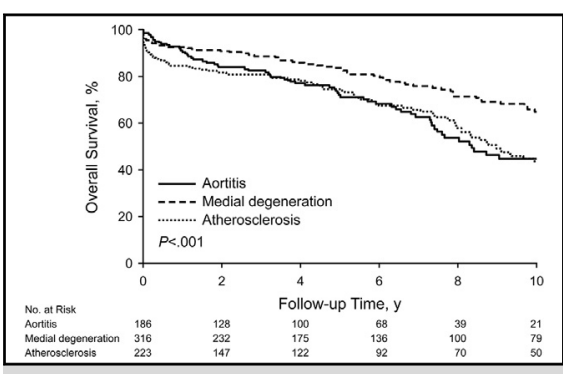

Overall survival differed significantly between groups.

Central Message

Patients with aneurysms and aortitis have greater risk for reoperation $(21 \%)$ than do those with medial degeneration $(11 \%)$ or atherosclerosis (19\%).

\section{Perspective}

This study describes outcomes of patients who were found to have aortitis at the time of excision of ascending aortic aneurysms. Compared with patients who have medial degeneration or atherosclerosis, patients with aortitis have an increased risk of aortic reoperation. Precise pathologic diagnosis of ascending aortic aneurysms may be helpful in stratifying prognosis and treatment.

See Editorial Commentary page 595.
Inflammation of the aortic wall is an important cause of aortic aneurysms, and noninfectious aortitis is being identified with increasing frequency in patients undergoing elective replacement of the ascending aorta. ${ }^{1}$ Of the patients who are found to have aortitis, $96 \%$ have no associated systemic illness. ${ }^{2}$ In surgical series, the prevalence of noninfectious aortitis among patients with aortic aneurysms ranges from $4.4 \%$ to $8.4 \%$, yet few data are available to guide surgical and postoperative management. ${ }^{1,2}$

\footnotetext{
From the Divisions of ${ }^{\mathrm{a} C a r d i o v a s c u l a r}$ Surgery, ${ }^{\mathrm{b}}$ Anatomic Pathology, and ${ }^{\mathrm{c}}$ Biostatistics and Informatics, Mayo Clinic, Rochester, Minn.

Read at the American Association for Thoracic Surgery Aortic Symposium, New York, New York, April 24-25, 2014.

Received for publication Dec 30, 2014; revisions received June 5, 2015; accepted for publication June 16, 2015; available ahead of print July 15, 2015.

Address for reprints: Hartzell V. Schaff, MD, Division of Cardiovascular Surgery,

Mayo Clinic, 200 First St SW, Rochester, MN 55905 (E-mail: schaff@ mayo.edu). $0022-5223 / \$ 36.00$

Copyright (c) 2015 by The American Association for Thoracic Surgery

http://dx.doi.org/10.1016/j.jtcvs.2015.06.027
}

Previous studies of surgical treatment of patients with aortic aneurysms secondary to aortitis have focused on the extent of aortic involvement and surgical methods, including the need for aortic valve replacement. ${ }^{3}$ However, no large studies have been done of late outcomes of ascending aneurysm repair in patients who have aortitis, compared with patients who have medial degeneration or atherosclerosis. The purpose of this study was to determine whether the outcome of repair of ascending aortic aneurysms, in patients who have noninfectious aortitis, differs from the outcome of operation in patients with aneurysms caused by atherosclerosis or medial degeneration.

\section{METHODS \\ Cohort Selection}

After obtaining approval from the Mayo Clinic Institutional Review Board, we reviewed all patients aged $>18$ years who underwent surgical replacement of the ascending aorta between January 1, 1955, and December 31, 2012, at Mayo Clinic in Rochester, Minn. Patients were 


\section{Abbreviations and Acronyms \\ $\mathrm{CI}=$ confidence interval \\ $\mathrm{HR}=$ hazard ratio}

excluded if they did not give permission for research or did not have available pathology reports. Patients were additionally excluded if they had aneurysms secondary to aortic dissection, heritable connective tissue disease (eg, Marfan syndrome or Ehlers-Danlos syndrome), infectious aortitis, or congenital heart disease, including bicuspid aortic valve. Patients with Takayasu arteritis were excluded because they are generally younger and have characteristic clinical presentations and associated obstructive aortic disease (Figure 1).

Preoperative and postoperative clinical data were obtained from the patients' electronic health records and a cardiovascular surgical database. Additional follow-up data were obtained from patient and physician correspondence, and from questionnaires sent to patients $1,3,5,10,15$, and 20 years after their operation.

\section{Histopathologic Evaluation}

Patients who met study criteria were divided into 3 groups, according to the cause of their ascending aortic aneurysm as assessed via a histologic examination of the surgical specimen, conducted by a cardiovascular pathologist. The resected ascending aortic wall was fixed in $10 \%$ neutral buffered formalin, embedded in paraffin, cut at a thickness of $4 \mu \mathrm{m}$, and stained with hematoxylin-eosin and Verhoeff-van Gieson. Typically, 6 to 8 transmural sections were evaluated per patient.

Presence of aortitis was defined as medial inflammation, with associated medial damage. The character of the inflammatory infiltrate was noted as well-specifically, whether giant cells were present. Presence of medial degeneration was defined as fragmentation of elastic lamella, with associated mucopolysaccharide deposition. Presence of atherosclerosis was defined as intimal collections of foamy histiocytes and cholesterol, with or without associated inflammation and associated subjacent secondary medial change. The presence of any aortitis prompted classification as such. Those without aortitis were dichotomized into diagnosis categories of medial degeneration or atherosclerosis, depending on which process was most evident.

\section{Statistical Analysis}

Categoric data were summarized as frequency and percentage of samples; continuous data were summarized as mean \pm SD. Categoric data for the groups were compared, using $\chi^{2}$ analysis or the Fisher exact test; continuous data were compared using standard ANOVA or KruskalWallis ANOVA, as appropriate. The Kaplan-Meier product-limit method was used to draw survival curves and calculate 5-year and 10-year survival, and estimates for freedom from reoperation.

Cox regression models were used to find the univariate and multivariate predictors of overall mortality and time to aortic operation. The multivariate models included variables that were significant on univariate analysis $(P<.05)$, with model selection using the stepwise method (backward and forward methods resulted in the same model). All statistical tests were 2-sided.

\section{RESULTS}

Between 1955 and 2012, a total of 3177 patients underwent ascending aorta replacement. Of these patients, 735 met criteria for the study and were divided into 3 groups according to aortic pathology (Figure 2). Inflammatory, noninfectious aortitis was identified in 186 patients; medial degeneration was present in 317 patients; and atherosclerosis was the pathologic finding in 232 patients.

\section{Demographics}

Among patients with noninfectious, inflammatory aortitis, the mean age was $71 \pm 11$ years, and $35 \%$ of the patients (66 of 186) were men. These demographic data differed $(P<.001)$ from data for patients who had medial degeneration (mean age: $64 \pm 14$ years; $69 \%$ men [219 of 317], $P<.001)$ and for patients with atherosclerosis (mean age: $66 \pm 11$ years; $61 \%$ men [141 of 232], $P<.001$ ). Other vascular conditions, such as occlusive peripheral vascular disease $(P<.001)$, coronary artery disease $(P=.003)$, and stroke $(P=.010)$, were significantly less prevalent in patients with medial degeneration, compared with patients who had noninfectious aortitis or atherosclerosis (Table 1). Among the 186 patients with noninfectious aortitis, giant cells were identified in 138 patients $(74 \%)$, and lymphoplasmacytic infiltrates without giant cells were observed in 48 patients $(26 \%)$.

Among patients who had preoperative echocardiography $(\mathrm{n}=348)$, the mean diameter of the midascending aorta was $57.3 \mathrm{~mm}$ in the group with noninfectious aortitis, $51.5 \mathrm{~mm}$ in the medial degeneration group $(P<.001)$, and $52.7 \mathrm{~mm}$ in the atherosclerosis group $(P<.001)$. Moderate-to-severe aortic valve regurgitation was diagnosed preoperatively in $56 \%$ (103 of 183) of patients with aortitis, 63\% (190 of $300)$ of patients with medial degeneration, and $47 \%$ (92 of 195) of patients with atherosclerosis $(P<.001$ for all 3 groups; $P<.010$ for comparison of the medial degeneration group with the atherosclerosis group).

\section{Aortic Valve}

Concomitant aortic valve procedures were performed in $58 \%$ (107 of 186) of patients with noninfectious aortitis, $77 \%$ (243 of 317) of patients with medial degeneration $(P<.001)$, and $59 \%$ (137 of 232$)$ of patients with atherosclerosis $(P<.753)$. Among patients who underwent concomitant aortic valve operations, aortic valve-sparing procedures were used relatively more often in patients with noninfectious aortitis $(40 \% ; 42$ of 106$)$ than in patients

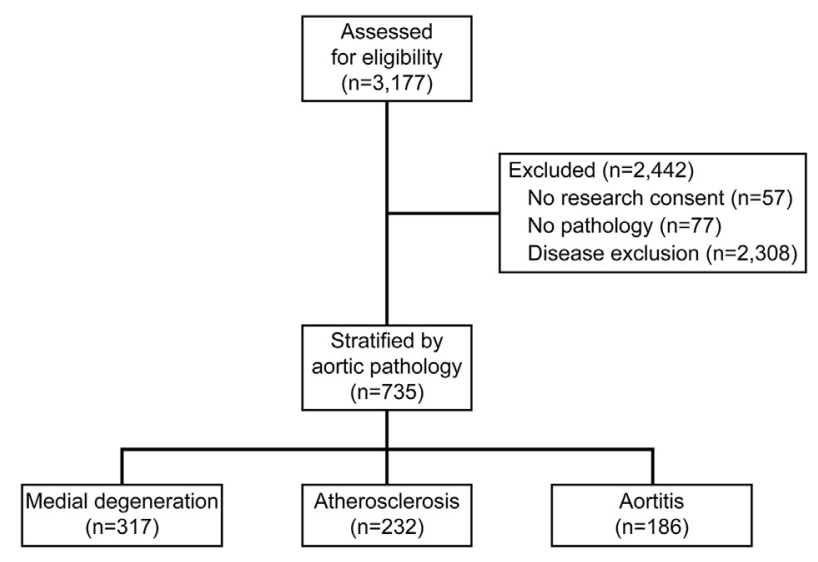

FIGURE 1. Patient selection and stratification. 


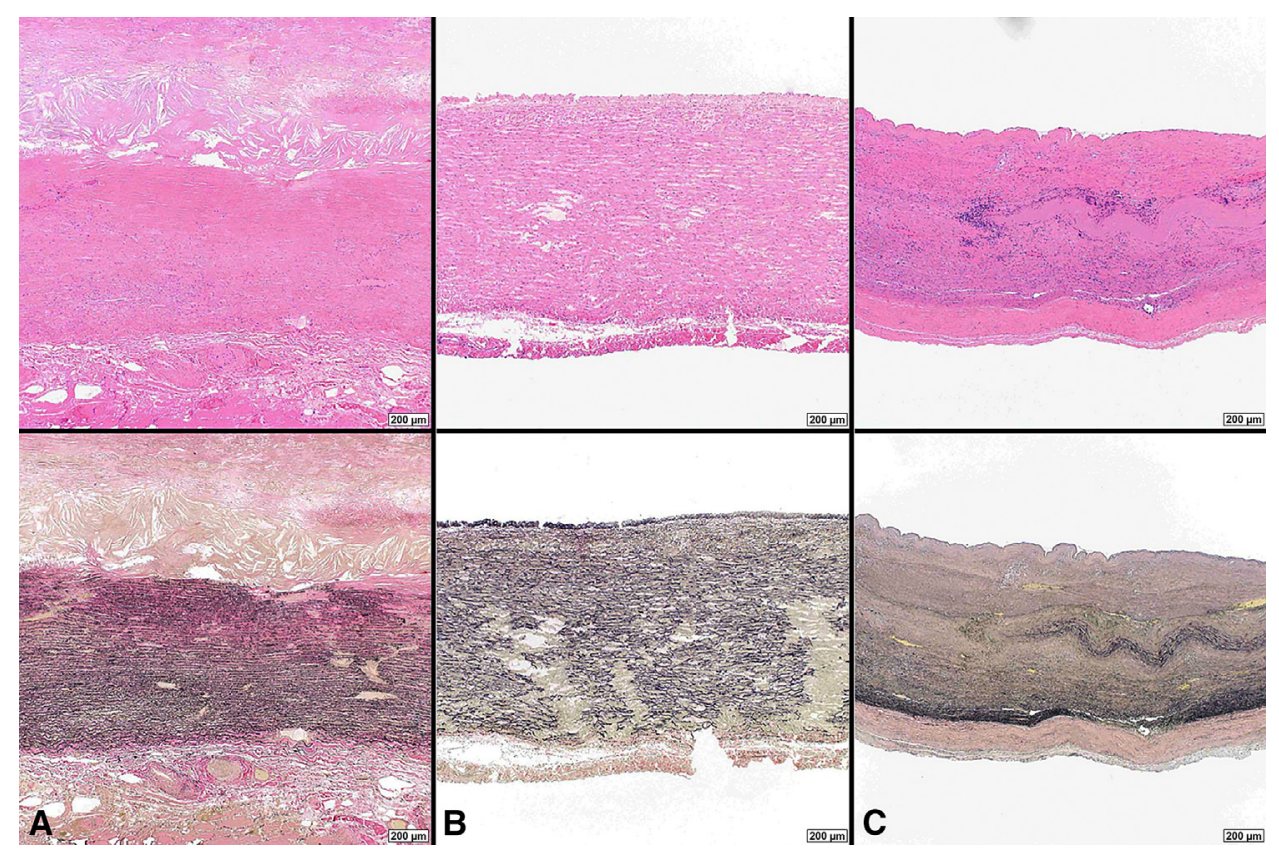

FIGURE 2. Aortic pathology: A, Aortic atherosclerosis is characterized by intimal plaques containing cholesterol clefts and foam cells. The subjacent media show mild, secondary, degenerative changes. B, Medial degeneration is characterized by elastic membrane fragmentation and mucopolysaccharide deposition throughout the media. C, Giant cell aortitis is characterized by medial inflammation with multinucleated giant cells imparting a moth-eaten appearance to the aortic media (top panels: hematoxylin-eosin; bottom panels: Verhoeff-van Gieson; original magnification $\times 40$ for all).

with medial degeneration $(33 \% ; 81$ of 243$)(P<.285)$ and atherosclerosis $(28 \% ; 38$ of 137$)(P=.057)$.

Among patients with aortitis who underwent aortic valve-sparing operations, 5- and 10-year estimates of risk of aortic valve reoperation were $15 \%$ and $22 \%$, respectively, compared with $10 \%$ and $10 \%$ for patients with aortitis who had aortic valve replacement $(P=.240)$. In patients with medial degeneration and patients with atherosclerosis who underwent valve-sparing operations, estimates of cumulative risk of aortic valve reoperation were $5 \%$ and $3 \%$ (at 5 years), and $11 \%$ and $22 \%$ (at 10 years), respectively $(P=.072)$. Thirty-two patients $(17 \%)$ in the aortitis group required total root replacement, compared with 108 patients $(34 \%)$ in the medial degeneration group, and 38 patients $(16 \%)$ in the atherosclerosis group.

\section{Extent of Aortic Resection}

The extent of aortic resection differed in the 3 histopathologically defined groups. Approximately one-half of the patients $(48 \%$; 90 of 186) who had aortitis had extensive aortic resection involving the aortic arch (complete or hemiarch), compared with $16 \%$ (52 of 317) $(P<.001)$ of patients with medial degeneration, and $35 \%$ (82 of 232) $(P=.007)$ of patients with atherosclerosis. Hemiarch replacements were performed in 59 patients $(32 \%)$ with aortitis, 44 patients $(14 \%)$ with medial degeneration, and 53 patients $(23 \%)$ with atherosclerosis; total arch replacements were performed in 17\% (31 of 186), 3\% (8 of
317 ), and $13 \%$ (29 of 232) of patients in the 3 groups, respectively.

\section{Aortic Reoperation and Survival}

Cumulative risk of aortic reoperation at 5 and 10 years, respectively, was $13 \%$ (95\% confidence interval [CI]: $7 \%-19 \%)$ and $21 \%(95 \%$ CI: $11 \%-30 \%)$ for patients with aortitis; 6\% (95\% CI: 3\%-10\%) and 11\% (95\% CI: $5 \%-16 \%$ ) for patients with medial degeneration; and 9\% (95\% CI: 5\%-14\%) and 19\% (95\% CI: $11 \%-27 \%)$ for patients with atherosclerosis $(P=.025)$ (Figure 3). These include aortic reoperations as the second stage of an elephant trunk procedure. Most reoperations were for distal progressive disease, except for 1 patient with aortitis who required total arch replacement after a hemiarch procedure initially. However, when predictors for aortic reoperation were examined in multivariate analysis, the cause of aortic aneurysm was found to not be a significant variable. The predictors of aortic reoperation were peripheral vascular disease (hazard ratio [HR]: 2.290; 95\% CI: $1.377-3.802 ; P=.001)$ and extended aortic arch procedures at the primary operation (HR: $3.510 ; 95 \%$ CI: 2.083-5.930; $P<.001$ ) (Table 2).

Among all patients, mortality was influenced by associated cardiac disease and the era of operation. Risk of operative death (in the hospital or $<30$ days postoperatively) was $3 \%$ for patients undergoing isolated aortic operations. Operative mortality for ascending aortic replacement with or 
TABLE 1. Demographics and operative details

\begin{tabular}{|c|c|c|c|c|}
\hline Variable & Aortitis $(n=186)$ & Medial degeneration $(n=317)$ & Atherosclerosis $(\mathrm{n}=\mathbf{2 3 2})$ & $P$ value \\
\hline Age (y) & $71 \pm 11$ & $64 \pm 14$ & $66 \pm 11$ & $<.001$ \\
\hline Gender, male & $66(35)$ & $219(69)$ & $141(61)$ & .001 \\
\hline Smoking history & $99(53)$ & $157(50)$ & $119(51)$ & .590 \\
\hline COPD & $36(19)$ & $29(9)$ & $40(17)$ & .002 \\
\hline Diabetes mellitus & $18(10)$ & $22(7)$ & $24(10)$ & .310 \\
\hline Hypercholesterolemia & $109(59)$ & $149(47)$ & $106(46)$ & .010 \\
\hline Hypertension & $136(73)$ & $214(68)$ & $137(59)$ & .005 \\
\hline PVD & $37(20)$ & $39(12)$ & $58(25)$ & $<.001$ \\
\hline CAD & $81(44)$ & $99(31)$ & $96(41)$ & .003 \\
\hline Stroke & $27(15)$ & $23(7)$ & $31(13)$ & .010 \\
\hline Arrhythmia & $32(17)$ & $64(20)$ & $39(17)$ & .580 \\
\hline NYHA class & & & & .009 \\
\hline I or II & $113(63)$ & $171(59)$ & $88(48)$ & \\
\hline III or IV & $65(37)$ & $117(41)$ & $94(52)$ & \\
\hline \multicolumn{5}{|l|}{ Aortic dimensions (mm) } \\
\hline Annulus diameter & $23.5 \pm 2.9$ & $24.6 \pm 3.8$ & $24.6 \pm 2.6$ & .020 \\
\hline Sinus of Valsalva & $40.1 \pm 7.3$ & $46.3 \pm 8.6$ & $43.4 \pm 7.0$ & $<.001$ \\
\hline Sinotubular junction & $37.0 \pm 8.8$ & $41.2 \pm 7.7$ & $39.6 \pm 8.0$ & $<.001$ \\
\hline Midascending aorta & $57.3 \pm 9.7$ & $51.5 \pm 9.8$ & $52.7 \pm 9.2$ & $<.001$ \\
\hline Arch & $35.8 \pm 7.4$ & $34.6 \pm 6.6$ & $37.7 \pm 8.9$ & .110 \\
\hline Aortic insufficiency, moderate to severe $(\%)$ & 56 & 63 & 47 & .010 \\
\hline Aortic stenosis $(\%)$ & 5 & 14 & 20 & $<.001$ \\
\hline $\mathrm{EF}(\%)$ & $58.5 \pm 11.3$ & $57.2 \pm 10.9$ & $56.5 \pm 11.5$ & .230 \\
\hline Aortic valve procedure & & & & $<.001$ \\
\hline Replacement & $64(60)$ & $163(51)$ & $99(44)$ & \\
\hline Sparing & $42(40)$ & $81(33)$ & $38(28)$ & \\
\hline Arch procedure & $90(48)$ & $52(16)$ & $82(35)$ & $<.001$ \\
\hline Hemiarch & $59(32)$ & $44(14)$ & $53(23)$ & \\
\hline Total arch & $5(3)$ & $2(0.6)$ & $9(4)$ & \\
\hline Total arch and elephant trunk & $21(11)$ & $5(1.6)$ & $14(6)$ & \\
\hline Total arch and descending & $5(3)$ & $1(0.3)$ & $6(3)$ & \\
\hline Concomitant MVR & $10(5)$ & $30(9)$ & $8(4)$ & .020 \\
\hline Concomitant CABG & $61(33)$ & $77(24)$ & $72(32)$ & .850 \\
\hline CPB time (min) & $132 \pm 54$ & $128 \pm 58$ & $134 \pm 58$ & .260 \\
\hline Crossclamp time (min) & $84 \pm 39$ & $92 \pm 43$ & $84 \pm 38$ & .090 \\
\hline Circulatory arrest & $114(82)$ & $71(37)$ & $95(51)$ & $<.001$ \\
\hline Circulatory arrest time (min) & $21 \pm 11.9$ & $19 \pm 8.3$ & $23 \pm 13.5$ & .600 \\
\hline Operative mortality & $4(2)$ & $15(5)$ & $18(8)$ & .020 \\
\hline
\end{tabular}

without concomitant arch replacement, aortic valve replacement, or coronary artery bypass grafting decreased during the study period and was $2 \%$ during and after 2010 $(P<.001)$ (Figure 4). Overall operative mortality was higher in the atherosclerosis group $(8 \%)$ than in the noninfectious aortitis group $(2 \% ; P=.008)$ and the medial degeneration group $(5 \% ; P=.023)$, but this was likely related to the era of operation. Before 1990, atherosclerotic aneurysms $(30 \%)$ were identified more frequently, compared with aortitis $(4 \%)$ and medial degeneration (13\%).

Overall survival at 5 years was $72 \%$ (95\% CI: $64 \%$ $79 \%$ ) for patients with aortitis; $84 \%$ (95\% CI: $80 \%$ -
$89 \%$ ) for patients with medial degeneration; and $74 \%$ (95\% CI: $68 \%-81 \%$ ) for patients with atherosclerosis; survival at 10 years was $45 \%$ (95\% CI: $36 \%-57 \%$ ); $66 \%$ (95\% CI: $59 \%-73 \%$ ); and 45\% (95\% CI: $37 \%-54 \%$ ) $(P<.001)$, respectively (Figure 5). In multivariate analysis, predictors of overall mortality included older age at operation (HR: $1.060 ; 95 \%$ CI: 1.046-1.077; $P<.001$ ); chronic obstructive pulmonary disease (HR: $1.560 ; 95 \%$ CI: 1.136-2.136; $P=.006$ ); concomitant coronary artery bypass grafting (HR: 1.980; 95\% CI: 1.520-2.600; $P<.001)$; and more extensive aortic resection, as reflected by the use of circulatory arrest during repair (HR: 1.500; 


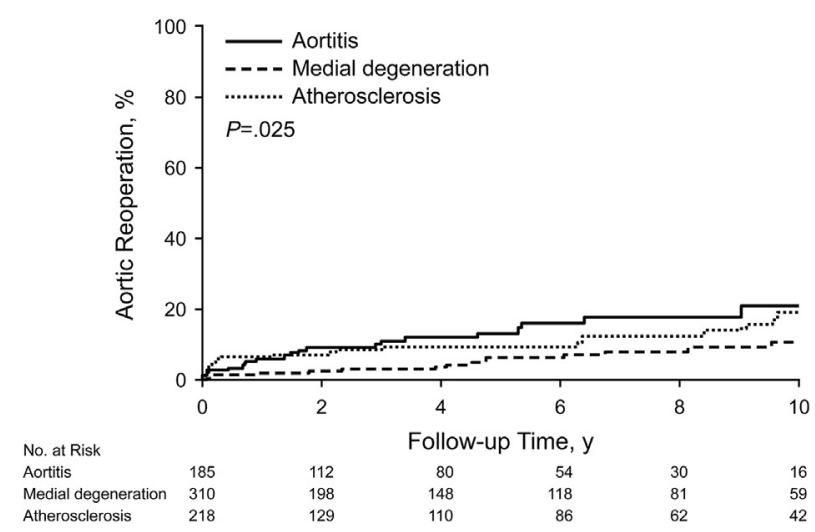

FIGURE 3. Risk of aortic reoperation: Cumulative risk of aortic reoperation at 5 years was $13 \%$ for patients with aortitis; $6 \%$ for patients with medial degeneration; and $9 \%$ for patients with atherosclerosis; for the respective groups, the 10-year cumulative risk of aortic reoperation was $21 \%(95 \%$ CI: $11 \%-30 \%)$; $11 \%$ (95\% CI: $5 \%-16 \%)$; and $19 \%(95 \%$ CI: $11 \%-27 \%)(P=.025)$.

95\% CI: $1.148-1.960 ; P=.003)$. After 1990 , overall survival was improved with operation (HR: $0.620 ; 95 \% \mathrm{CI}$ : $0.444-0.863 ; P<.001)$. In pairwise comparison in the multivariate analysis, patients with medial degeneration (HR: 0.720; 95\% CI: $0.523-0.984 ; P=.040$ ) had a lower risk of death than patients with aortitis, but outcomes with aortitis versus atherosclerosis were not significantly different (HR: 0.960; 95\% CI: 0.705-1.313; $P=.809$ ) (Table 3).

\section{DISCUSSION}

The diagnosis of aortitis may be suspected preoperatively in patients with systemic inflammatory disorders or documented arteritis (eg, temporal arteritis). But, as found in this study and other surgical series, the diagnosis of aortitis in patients with ascending aortic aneurysms is usually made by pathologic examination of the aortic specimen, and patients frequently have few or no symptoms or signs of rheumatologic disorders preoperatively (2). Important clinical features of patients with noninfectious aortitis in our study were older age, high prevalence of female gender, and frequent involvement of the arch portion of the aorta.

In the present study, the diagnosis of aortitis was based on histopathologic evaluations of the resected specimen at operation, and findings in these patients were distinct from other causes, medial degeneration, and atherosclerosis. ${ }^{1,4}$ These findings underscore the importance of

TABLE 2. Multivariate analysis of factors affecting aortic reoperation

\begin{tabular}{lcr}
\hline \multicolumn{1}{c}{ Variable } & Hazard ratio $(\mathbf{9 5} \% \mathbf{C I})$ & $\boldsymbol{P}$ value \\
\hline Medial degeneration & $0.680(0.360-1.295)$ & .242 \\
Atherosclerosis & $0.850(0.471-1.526)$ & .582 \\
Peripheral vascular disease & $2.290(1.377-3.802)$ & .001 \\
Arch resection & $3.510(2.083-5.930)$ & $<.001$ \\
\hline
\end{tabular}

CI, Confidence interval.

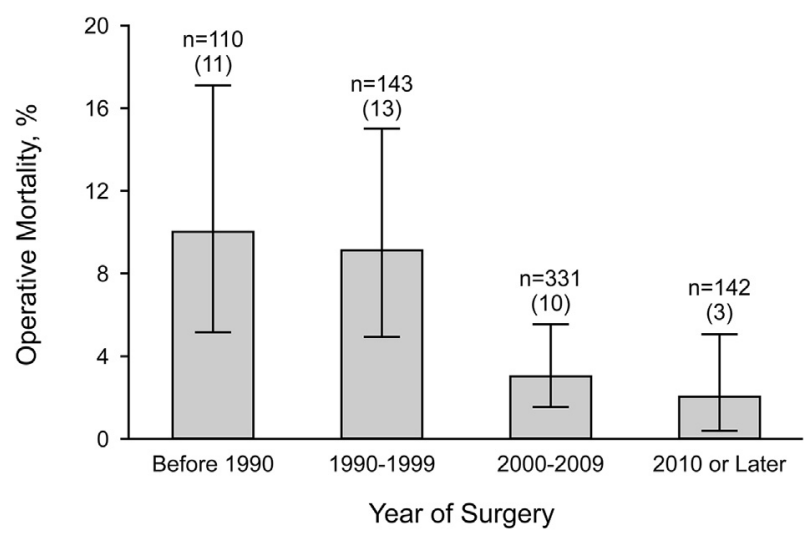

FIGURE 4. Operative mortality: Risk of death for repair of ascending aortic aneurysm (within 30 days postoperatively) varied by decade of operation. Mortality was 10\% before 1990; 9\% between 1990 and 1999; 3\% between 2000 and 2009; and 2\% during and after 2010. Sample sizes are shown as number of patients (number of events). Error bars indicate $95 \%$ CIs.

routine histopathologic assessment of surgically resected aortic specimens: The underlying pathology of aortic disease may guide patient care and screening. The pathologic identification of inflammatory aortitis can lead to the diagnosis of systemic diseases, such as giant cell arteritis, in some patients. ${ }^{5,6}$ Even if patients with inflammatory aortitis do not have an identifiable systemic illness (eg, lupus and giant cell arteritis), they do have an increased risk of peripheral vascular disease.

We found differences in the extent of aortic resection and operative methods among patients in the 3 distinct pathology groups. Almost half of the patients with noninfectious aortitis underwent hemiarch or total arch replacement at the first surgical intervention. The need for these extensive procedures reflects more-extensive involvement of the aorta beyond the ascending portion and is consistent with our previous clinical studies.

Fewer patients with noninfectious aortitis required aortic valve procedures (aortic valve replacement and aortic valve-sparing operations) at the primary operation, compared with patients with medial degeneration or atherosclerosis. Among patients with aortitis who underwent aortic valve procedures, $40 \%$ had an aortic valve-sparing operation. Other reports have suggested that valve-sparing operations can be performed frequently in patients with aneurysms secondary to aortitis.

In a clinical review, Mennander and colleagues ${ }^{3}$ found that $61 \%$ of patients with aortic valve regurgitation and aortitis were able to undergo a valve-sparing procedure; often, aortic valve regurgitation was secondary to dilatation of the sinotubular junction, but information on the long-term durability of valve-sparing procedures in this population is sparse. Data from the present study show that valvesparing operations are reasonably durable in patients with aortitis (10-year risk of aortic valve reoperation, $21 \%)$. 


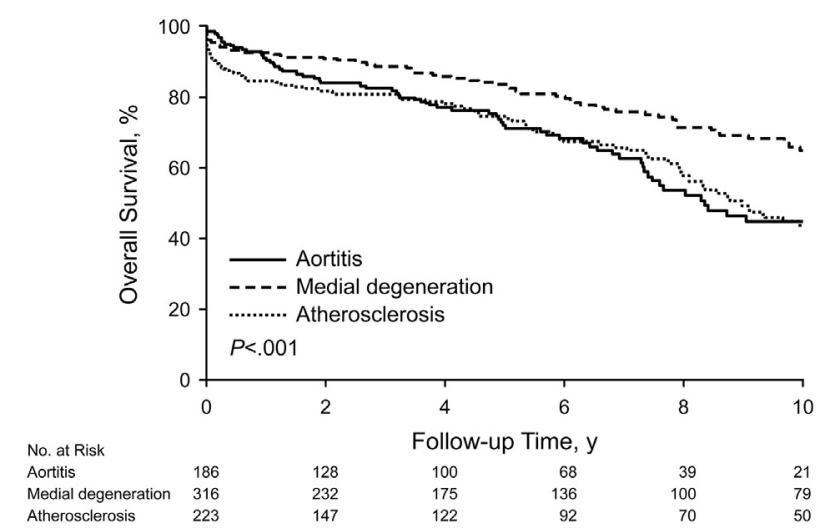

FIGURE 5. Overall survival differed significantly among the groups. At 5 years, survival was $72 \%$ for patients with aortitis, $84 \%$ for patients with medial degeneration, and $74 \%$ for patients with atherosclerosis; for the respective groups, 10-year survival was $45 \%$ (95\% CI: 36\%-57\%); 66\% (95\% CI: $59 \%-73 \%)$; and $45 \%(95 \%$ CI: 37\%-54\%) $(P<.001)$.

Risk of reoperation on the aorta did seem to differ in patients with aortitis, compared with patients who had other disorders. However, pathologic group was not a risk factor in multivariate analysis. Independent predictors of aortic reoperation were peripheral vascular disease and aortic arch procedures. Our study and others show that patients with aortitis often undergo more-extended aortic arch procedures. ${ }^{3,8}$ This likely reflects more-extensive aortic involvement with the disease and suggests the need for close postoperative surveillance for additional aneurysm formation.

Presence of aortitis does not seem to increase the early risk of ascending aortic aneurysm repair. In this study, operative mortality (in the hospital or $<30$ days postoperatively) was $2 \%$ in the aortitis patients, and this finding is consistent with earlier reports from Mayo Clinic. ${ }^{3,8}$ Overall survival postoperatively was reduced $(45 \%$ at 10 years) among patients with aortitis, compared with patients who had medial degeneration, but it was similar to that of patients who had aneurysms caused by atherosclerosis. The explanation for poorer survival of aortitis patients is unclear, but it may relate to progression of the underlying inflammatory disease, which can lead to renal dysfunction. ${ }^{9}$ In this study, we do not have information on

TABLE 3. Multivariate analysis of factors affecting overall survival

\begin{tabular}{lcr}
\hline \multicolumn{1}{c}{ Variable } & Hazard ratio $(\mathbf{9 5} \% \mathbf{C I})$ & $\boldsymbol{P}$ value \\
\hline Medial degeneration & $0.720(0.523-0.984)$ & .040 \\
Atherosclerosis & $0.960(0.705-1.313)$ & .809 \\
Age & $1.060(1.046-1.077)$ & $<.001$ \\
Surgical year 1990-1999 & $0.620(0.444-0.863)$ & .005 \\
Surgical year 2000-2009 & $0.360(0.246-0.525)$ & $<.001$ \\
Surgical year 2010 or later & $0.120(0.045-0.303)$ & $<.001$ \\
Presence of COPD & $1.560(1.136-2.136)$ & .006 \\
Combined CABG & $1.980(1.518-2.595)$ & $<.001$ \\
Circulatory arrest & $1.500(1.148-1.960)$ & .003 \\
\hline
\end{tabular}

$\overline{C O P D}$, Chronic obstructive pulmonary disease; $C A B G$, coronary artery bypass grafting; $C I$, confidence interval. the effectiveness of treatment with anti-inflammatory medications such as corticosteroids.

\section{Limitations}

Although this investigation examined late outcomes of mortality and aortic reoperation, we analyzed only preoperative and operative factors that might be associated with these end points. In this analysis, we did not have information on postoperative treatment, including antiinflammatory therapy. We had limited preoperative echocardiographic data availability. Because we excluded patients who did not have pathologic analysis, we limited our cohort size and perhaps the generalizability of our data. This study was retrospective, with the usual limitations related to the design, but we did have many patients, and pathologic confirmation for comparison groups.

\section{CONCLUSIONS}

Patients undergoing repair of ascending aortic aneurysms secondary to noninfectious inflammatory aortitis have low early $(<1$ year) mortality but an increased late $(>5$ year) risk of death and aortic reoperation compared with patients who have ascending aortic aneurysms secondary to medial degeneration. Postoperative medical management and screening measures and their effects on late outcomes must be studied.

\section{Conflict of Interest Statement}

Dr Suri reports consulting fees for Sorin and Abbott and grant support from Edwards, Sorin, and St. Jude. All other authors have nothing to disclose with regard to commercial support.

\section{References}

1. Maleszewski JJ. Inflammatory ascending aortic disease: perspectives from pathology. J Thorac Cardiovasc Surg. 2015;149:S176-83.

2. Rojo-Leyva F, Ratliff NB, Cosgrove DM III, Hoffman GS. Study of 52 patients with idiopathic aortitis from a cohort of 1,204 surgical cases. Arthritis Rheum. 2000;43:901-7.

3. Mennander AA, Miller DV, Liang KP, Warrington KJ, Connolly HM, Schaff HV, et al. Surgical management of ascending aortic aneurysm due to non-infectious aortitis. Scand Cardiovasc J. 2008;42:417-24.

4. Homme JL, Aubry MC, Edwards WD, Bagniewski SM, Shane Pankratz V, Kral CA, et al. Surgical pathology of the ascending aorta: a clinicopathologic study of 513 cases. Am J Surg Pathol. 2006;30:1159-68.

5. Agard C, Ponge T, Fradet G, Baron O, Sagan C, Masseau A, et al. Giant cell arteritis presenting with aortic dissection: two cases and review of the literature. Scand J Rheumatol. 2006;35:233-6.

6. Espitia O, Neel A, Leux C, Connault J, Espitia-Thibault A, Ponge T, et al. Giant cell arteritis with or without aortitis at diagnosis: a retrospective study of 22 patients with longterm followup. J Rheumatol. 2012;39:2157-62.

7. Liang KP, Chowdhary VR, Michet CJ, Miller DV, Sundt TM, Connolly HM, et al. Noninfectious ascending aortitis: a case series of 64 patients. J Rheumatol. 2009; 36:2290-7.

8. Zehr KJ, Mathur A, Orszulak TA, Mullany CJ, Schaff HV. Surgical treatment of ascending aortic aneurysms in patients with giant cell aortitis. Ann Thorac Surg. 2005;79:1512-7.

9. Gornik HL, Creager MA. Aortitis. Circulation. 2008;117:3039-51.

Key Words: aortic aneurysm, aortic repair, aortitis 